\title{
Global Weak Solutions of the Navier-Stokes Equations with Nonhomogeneous Boundary Data and Divergence
}

\author{
R. FARWIG $(*)-$ H. Kozono $(* *)-H . \operatorname{SoHR}(* * *)$
}

ABSTRACT - Consider a smooth bounded domain $\Omega \subseteq \mathbb{R}^{3}$ with boundary $\partial \Omega$, a time interval $[0, T), 0<T \leq \infty$, and the Navier-Stokes system in $[0, T) \times \Omega$, with initial value $u_{0} \in L_{\sigma}^{2}(\Omega)$ and external force $f=\operatorname{div} F, F \in L^{2}\left(0, T ; L^{2}(\Omega)\right)$. Our aim is to extend the well-known class of Leray-Hopf weak solutions $u$ satisfying $\left.u\right|_{\partial \Omega}=0, \operatorname{div} u=0$ to the more general class of Leray-Hopf type weak solutions $u$ with general data $\left.u\right|_{\partial \Omega}=g$, div $u=k$ satisfying a certain energy inequality. Our method rests on a perturbation argument writing $u$ in the form $u=v+E$ with some vector field $E$ in $[0, T) \times \Omega$ satisfying the (linear) Stokes system with $f=0$ and nonhomogeneous data. This reduces the general system to a perturbed Navier-Stokes system with homogeneous data, containing an additional perturbation term. Using arguments as for the usual Navier-Stokes system we get the existence of global weak solutions for the more general system.

\section{Introduction and main results.}

Let $\Omega \subseteq \mathbb{R}^{3}$ be a bounded domain with boundary $\partial \Omega$ of class $C^{2,1}$, and let $[0, T), 0<T \leq \infty$, be a time interval. We consider in $[0, T) \times \Omega$, together with an associated pressure $p$, the following general Navier-Stokes system

$$
\begin{array}{rlrl}
u_{t}-\Delta u+u \cdot \nabla u+\nabla p & =f, & & \operatorname{div} u=k \\
\left.u\right|_{\partial \Omega} & =g, & \left.u\right|_{t=0}=u_{0}
\end{array}
$$

with given data $f, k, g, u_{0}$.

(*) Indirizzo dell'A.: Department of Mathematics, Darmstadt University of Technology, 64289 Darmstadt, Germany, and Center of Smart Interfaces (CSI), 64287 Darmstadt, Germany.

E-mail: farwig@mathematik.tu-darmstadt.de

(**) Indirizzo dell'A.: Mathematical Institute, Tôhoku University, Sendai, 980-8578 Japan.

E-mail: kozono@math.tohoku.ac.jp

(***) Indirizzo dell'A.: Faculty of Electrical Engineering, Informatics and Mathematics, University of Paderborn, 33098 Paderborn, Germany.

E-mail: hsohr@math.uni-paderborn.de 
First we have to give a precise characterization of this general system. To this aim, we shortly discuss our arguments to solve this system in the weak sense (without any smallness assumption on the data). Using a perturbation argument we write $u$ in the form

$$
u=v+E,
$$

and the initial value $u_{0}$ at time $t=0$ in the form

$$
u_{0}=v_{0}+E_{0} .
$$

Here $E$ is the solution of the (linear) Stokes system

$$
\begin{aligned}
E_{t}-\Delta E+\nabla h & =0, \quad \operatorname{div} E \\
\left.E\right|_{\partial \Omega} & =g,\left.\quad E\right|_{t=0}=E_{0}
\end{aligned}
$$

with some associated pressure $h$, and $v$ has the properties

$$
\begin{gathered}
v \in L_{\mathrm{loc}}^{\infty}\left([0, T) ; L_{\sigma}^{2}(\Omega)\right) \cap L_{\mathrm{loc}}^{2}\left([0, T) ; W_{0}^{1,2}(\Omega)\right), \\
v:[0, T) \mapsto L_{\sigma}^{2}(\Omega) \quad \text { is weakly continuous, } v_{t=0}=v_{0} .
\end{gathered}
$$

Inserting (1.2), (1.3) into the system (1.1) we obtain the modified system

$$
\begin{aligned}
& v_{t}-\Delta v+(v+E) \cdot \nabla(v+E)+\nabla p^{*}=f, \operatorname{div} v=0 \\
& \left.v\right|_{\partial \Omega}=0,\left.\quad v\right|_{t=0}=v_{0}
\end{aligned}
$$

with associated pressure $p^{*}=p-h$ and homogeneous conditions for $v$. Thus (1.6) can be called a perturbed Navier-Stokes system in $[0, T) \times \Omega$. This system reduces the general system (1.1) to a certain homogeneous system which contains an additional perturbation term in the form

$$
(v+E) \cdot \nabla(v+E)=v \cdot \nabla v+v \cdot \nabla E+E \cdot \nabla(v+E) .
$$

Therefore, the perturbed system (1.6) can be treated similarly as the usual Navier-Stokes system obtained from (1.6) with $E \equiv 0$.

In order to give a precise definition of the general system (1.1) we need the following steps:

First we develop the theory for the perturbed system (1.6) for data $f, v_{0}$ and a given vector field $E$, as general as possible. In the second step we consider the system (1.4) for general given data $k, g, E_{0}$ to obtain a vector field $E$ in such a way that $u=v+E$ with $v$ from (1.6) yields a well-defined solution of the general system (1.1) in the (Leray-Hopf type) weak sense.

Thus we start with the definition of a weak solution $v$ of (1.6) under rather weak assumptions on $E$ needed for the existence of such solutions. 
Definition 1.1. (Perturbed system). Suppose

$$
\begin{aligned}
& f=\operatorname{div} F \quad \text { with } \quad F=\left(F_{i, j}\right)_{i, j=1}^{3} \in L^{2}\left(0, T ; L^{2}(\Omega)\right), \\
& v_{0} \in L_{\sigma}^{2}(\Omega), \\
& E \in L^{s}\left(0, T ; L^{q}(\Omega)\right), \operatorname{div} E=k \in L^{4}\left(0, T ; L^{2}(\Omega)\right),
\end{aligned}
$$

with $4 \leq s<\infty, 4 \leq q<\infty, \frac{2}{s}+\frac{3}{q}=1$.

Then a vector field $v$ is called a weak solution of the perturbed system (1.6) in $[0, T) \times \Omega$ with data $f$, $v_{0}$ if the following conditions are satisfied:

a) For each finite $T^{*}, 0<T^{*} \leq T$,

$$
v \in L^{\infty}\left(0, T^{*} ; L_{\sigma}^{2}(\Omega)\right) \cap L^{2}\left(0, T^{*} ; W_{0}^{1,2}(\Omega)\right),
$$

b) for each test function $w \in C_{0}^{\infty}\left([0, T) ; C_{0, \sigma}^{\infty}(\Omega)\right)$,

$$
\begin{gathered}
-\left\langle v, w_{t}\right\rangle_{\Omega, T}+\langle\nabla v, \nabla w\rangle_{\Omega, T}-\langle(v+E)(v+E), \nabla w\rangle_{\Omega, T} \\
-\langle k(v+E), w\rangle_{\Omega, T}=\left\langle v_{0}, w(0)\right\rangle_{\Omega}-\langle F, \nabla w\rangle_{\Omega, T},
\end{gathered}
$$

c) for $0 \leq t<T$,

$$
\begin{gathered}
\frac{1}{2}\|v(t)\|_{2}^{2}+\int_{0}^{t}\|\nabla v\|_{2}^{2} d \tau \leq \frac{1}{2}\left\|v_{0}\right\|_{2}^{2}-\int_{0}^{t}\langle F, \nabla v\rangle_{\Omega} d \tau \\
+\int_{0}^{t}\langle(v+E) E, \nabla v\rangle_{\Omega} d \tau+\frac{1}{2} \int_{0}^{t}\langle k(v+2 E), v\rangle_{\Omega} d \tau,
\end{gathered}
$$

d) and

$$
v:[0, T) \rightarrow L_{\sigma}^{2}(\Omega) \text { is weakly continuous and } v(0)=v_{0} .
$$

In the classical case $E \equiv 0$ we obtain with (1.8)-(1.11) the usual (LerayHopf) weak solution $v$. As in this case the condition (1.11) already follows from the other conditions (1.8)-(1.10), after possibly a modification on a null set of $[0, T)$, see, e.g., [16, V, 1.6]. Here (1.11) is included for simplicity. The relation (1.9) and the energy inequality (1.10) are based on formal calculations as for $E \equiv 0$. The existence of an associated pressure $p^{*}$ such that

$$
v_{t}-\Delta v+(v+E) \cdot \nabla(v+E)+\nabla p^{*}=f
$$

in the sense of distributions in $(0, T) \times \Omega$ follows in the same way as for $E \equiv 0$.

In the next step we consider the linear system (1.4). A very general solution class for this system, sufficient for our purpose, has been devel- 
oped by the theory of so-called very weak solutions, see [1], [3, Sect. 4]. In particular, the boundary values $g$ are given in a general sense of distributions on $\partial \Omega$.

LEMma 1.2 (Linear system for $E$, [3]). Suppose

$$
\begin{aligned}
& k \in L^{s}\left(0, T ; L^{q^{*}}(\Omega)\right), \quad g \in L^{s}\left(0, T ; W^{-\frac{1}{q}, q}(\partial \Omega)\right), E_{0} \in L^{q}(\Omega), \\
& 4 \leq s<\infty, 4 \leq q<\infty, \frac{2}{s}+\frac{3}{q}=1, \frac{1}{q}=\frac{1}{q^{*}}-\frac{1}{3},
\end{aligned}
$$

satisfying the compatibility condition

$$
\int_{\Omega} k(t) d x=\int_{\partial \Omega} N \cdot g(t) d S \quad \text { for almost all } t \in[0, T),
$$

where $N=N(x)$ means the exterior normal vector at $x \in \partial \Omega$, and $\int_{\partial \Omega} \ldots d S$ the surface integral (in a generalized sense of distributions on $\partial \Omega$ ).

Then there exists a uniquely determined (very) weak solution

$$
E \in L^{s}\left(0, T ; L^{q}(\Omega)\right)
$$

of the system (1.4) in $[0, T) \times \Omega$ with data $k, g, E_{0}$ defined by the conditions:

a) For each $w \in C_{0}^{1}\left([0, T) ; C_{0, \sigma}^{2}(\bar{\Omega})\right)$,

$$
-\left\langle E, w_{t}\right\rangle_{\Omega, T}-\langle E, \Delta w\rangle_{\Omega, T}+\langle g, N \cdot \nabla w\rangle_{\Omega, T}=\left\langle E_{0}, w(0)\right\rangle_{\Omega},
$$

b) for almost all $t \in[0, T)$,

$$
\operatorname{div} E=k,\left.N \cdot E\right|_{\partial \Omega}=N \cdot g .
$$

Moreover, $E$ satisfies the estimate

$$
\left\|A_{q}^{-1} P_{q} E_{t}\right\|_{q, s ; \Omega, T}+\|E\|_{q, s ; \Omega, T} \leq C\left(\left\|E_{0}\right\|_{q}+\|k\|_{q^{*}, s ; \Omega, T}+\|g\|_{-\frac{1}{q} ; q, s ; \partial \Omega, T}\right)
$$

with constant $C=C(\Omega, T, q)>0$.

The trace $\left.E\right|_{\partial \Omega}=g$ is well-defined at $\partial \Omega$ for almost all $t \in[0, T)$, and the initial value condition $\left.E\right|_{t=0}=E_{0}$ is well-defined (modulo gradients) in the sense that $P_{q} E:[0, T) \rightarrow L_{\sigma}^{q}(\Omega)$ is weakly continuous satisfying

$$
\left.P_{q} E\right|_{t=0}=P_{q} E_{0} \text {. }
$$

Finally, there exists an associated pressure $h$ such that

$$
E_{t}-\Delta E+\nabla h=0
$$

holds in the sense of distributions in $(0, T) \times \Omega$. 
To obtain a precise definition for the general system (1.1) we have to combine Definition 1.1 and Lemma 1.2 as follows:

Definition 1.3. (General system). Let $k \in L^{s}\left(0, T ; L^{q^{*}}(\Omega)\right) \cap L^{4}\left(0, T ; L^{2}(\Omega)\right)$ with $s, q^{*}$ as in (1.13) and suppose that

$E$ is a very weak solution of the linear system (1.4) in

$[0, T) \times \Omega$ with data $k, g, E_{0}$ in the sense of Lemma 1.2,

and

$v$ is a weak solution of the perturbed system 1.6 in

$[0, T) \times \Omega$ in the sense of Definition 1.1 with data $f, v_{0}$

as in 1.7 .

Then the vector field $u=v+E$ is called a weak solution of the general system (1.1) in $[0, T) \times \Omega$ with data $f, k, g$ and initial value $u_{0}=v_{0}+E_{0}$. Thus it holds

$$
u_{t}-\Delta u+u \cdot \nabla u+\nabla p=f, \operatorname{div} u=k
$$

in the sense of distributions in $(0, T) \times \Omega$ with associated pressure $p=p^{*}+h, p^{*}$ as in (1.12), has in (1.20). Further,

$$
\left.u\right|_{\partial \Omega}=\left.v\right|_{\partial \Omega}+\left.E\right|_{\partial \Omega}=g
$$

is well-defined by $E_{\left.\right|_{\partial \Omega}}=g$, and the condition

$$
\left.u\right|_{t=0}=\left.v\right|_{t=0}+\left.E\right|_{t=0}=v_{0}+E_{0}=u_{0}
$$

is well-defined in the generalized sense modulo gradients by (1.19).

Therefore the general system (1.1) has a well-defined meaning for weak solutions $u$ in a generalized sense.

However, if we suppose in Definition 1.3 additionally the regularity properties

$$
\begin{aligned}
& k \in L^{s}\left(0, T ; W^{1, q}(\Omega)\right), k_{t} \in L^{s}\left(0, T ; L^{2}(\Omega)\right), \\
& g \in L^{s}\left(0, T ; W^{2-1 / q, q}(\partial \Omega)\right), g_{t} \in L^{s}\left(0, T ; W^{-\frac{1}{q}, q}(\partial \Omega)\right), \\
& E_{0} \in W^{2, q}(\Omega),
\end{aligned}
$$

and the compatibility conditions $\left.u_{0}\right|_{\partial \Omega}=\left.g\right|_{t=0}$, $\operatorname{div} u_{0}=\left.k\right|_{t=0}$, then the solution $E$ in Lemma 1.2 satisfies the regularity properties

$$
E \in L^{s}\left(0, T ; W^{2, q}(\Omega)\right), E_{t} \in L^{s}\left(0, T ; L^{q}(\Omega)\right), E \in C\left([0, T) ; L^{q}(\Omega)\right),
$$


and $\left.E\right|_{\partial \Omega}=g,\left.E\right|_{t=0}=E_{0}$ are well-defined in the usual sense, see [3, Corollary 5]. Further it holds $\nabla h \in L^{s}\left(0, T ; L^{q}(\Omega)\right)$ for the associated pressure $h$ in (1.20). Therefore, $u=v+E$ satisfies in this case the boundary condition $\left.u\right|_{\partial \Omega}=g$ and the initial condition $\left.u\right|_{t=0}=v_{0}+E_{0}$ in the usual (strong) sense.

The most difficult problem is the existence of a weak solution $v$ of the perturbed system (1.6). For this purpose we have to introduce, see (2.12) in Sect. 2, an approximate system of (1.6) for each $m \in \mathbb{N}$ which yields such a weak solution when passing to the limit $m \rightarrow \infty$. Then the existence of a weak solution $u=v+E$ of the general system (1.6) is an easy consequence.

This yields the following main result.

THEOREM 1.4 (Existence of general weak solutions).

a) Suppose

$$
\begin{aligned}
& f=\operatorname{div} F, F \in L^{2}\left(0, T ; L^{2}(\Omega)\right), v_{0} \in L_{\sigma}^{2}(\Omega), \\
& E \in L^{s}\left(0, T ; L^{q}(\Omega)\right), \operatorname{div} E=k \in L^{4}\left(0, T ; L^{2}(\Omega)\right), \\
& 4 \leq s<\infty, 4 \leq q<\infty, \frac{2}{s}+\frac{3}{q}=1 .
\end{aligned}
$$

Then there exists at least one weak solution $v$ of the perturbed system (1.6) in $[0, T) \times \Omega$ with data $f, v_{0}$ in the sense of Definition 1.1. The solution $v$ satisfies with some constant $C=C(\Omega)>0$ the energy estimate

$$
\begin{gathered}
\|v(t)\|_{2}^{2}+\int_{0}^{t}\|\nabla v\|_{2}^{2} d \tau \leq C\left(\left\|v_{0}\right\|_{2}^{2}+\int_{0}^{t}\|F\|_{2}^{2} d \tau+\int_{0}^{t}\|k\|_{2}^{4} d \tau\right. \\
\left.+\int_{0}^{t}\|E\|_{4}^{4} d \tau\right) \exp \left(C\|k\|_{2,4 ; t}^{4}+C\|E\|_{q, s ; t}^{s}\right)
\end{gathered}
$$

for each $0 \leq t<T$.

b) Suppose additionally

$$
\begin{aligned}
& k \in L^{s}\left(0, T ; L^{q^{*}}(\Omega)\right), g \in L^{s}\left(0, T ; W^{-\frac{1}{q}, q}(\partial \Omega)\right), E_{0} \in L^{q}(\Omega), \\
& \int_{\Omega} k d x=\int_{\partial \Omega} N \cdot g d S \text { for a.a. } t \in[0, T),
\end{aligned}
$$

and let $E$ be the very weak solution of the linear system $(1.4)$ in $[0, T) \times \Omega$ with data $k, g, E_{0}$ as in Lemma 1.2. Then $u=v+E$ is a weak solution of 
the general system (1.1) with data $f, k, g$ and initial value $u_{0}=v_{0}+E_{0}$ in the sense of Definition 1.3.

There are some partial results with nonhomogeneous smooth boundary conditions $\left.u\right|_{\partial \Omega}=g \neq 0$ based on an independent approach by Raymond [15]. For the case of weak solutions with constant in time nonzero boundary conditions $g$ see [4]. Further there are several independent results for smooth boundary values $\left.u\right|_{\partial \Omega}=g \neq 0$ in the context of strong solutions $u$ if $g$ or (equivalently) the time interval $[0, T)$ satisfy certain smallness conditions, see [1], [3], [6], [10]. Our existence result for weak solutions in Theorem 1.4 does not need any smallness condition, like for usual LerayHopf weak solutions. But, on the other hand, there is no uniqueness result as for local strong solutions.

A first result on global weak solutions with time-dependent boundary data (and $k=\operatorname{div} u=0$ ) can be found in [5]. In that paper, the authors consider general $s>2, q>3$ with $\frac{2}{s}+\frac{3}{q}=1$; however, in that case, $E$ has to
satisfy the assumptions

$$
E \in L^{s}\left(0, T ; L^{q}(\Omega)\right) \cap L^{4}\left(0, T ; L^{4}(\Omega)\right),
$$

which is automatically fulfilled in the present article, see Theorem 1.4. Moreover, in simply connected domains or under a further assumption on the boundary data $g$, the energy estimate (1.28) can be improved considerably.

\section{Preliminaries.}

First we recall some standard notations. Let $C_{0, \sigma}^{\infty}(\Omega)=\left\{w \in C_{0}^{\infty}(\Omega)\right.$; $\operatorname{div} w=0\}$ be the space of smooth, solenoidal and compactly supported vector fields. Then let $L_{\sigma}^{q}(\Omega)={\overline{C_{0, \sigma}^{\infty}(\Omega)}}^{\|\cdot\|_{q}}, 1<q<\infty$, where in general $\|\cdot\|_{q}$ denotes the norm of the Lebesgue space $L^{q}(\Omega), 1 \leq q \leq \infty$. Sobolev spaces are denoted by $W^{m, q}(\Omega)$ with norm $\|\cdot\|_{W^{m, q}}=\|\cdot\|_{m, q}, m \in \mathbb{N}, 1 \leq q \leq \infty$, and $W_{0}^{m, q}(\Omega)=\bar{C}_{0}^{\infty}(\Omega) \|^{\|, q}, 1 \leq q<\infty$. The trace space to $W^{1, q}(\Omega)$ is $W^{1-1 / q, q}(\partial \Omega), 1<q<\infty$, with norm $\|\cdot\|_{1-1 / q, q}$. Then the dual space to $W^{1-1 / q^{\prime}, q^{\prime}}(\partial \Omega)$, where $\frac{1}{q^{\prime}}+\frac{1}{q}=1$, is $W^{-1 / q, q}(\partial \Omega)$; the corresponding pairing is denoted by $\langle\cdot, \cdot\rangle_{\partial \Omega}$.

As spaces of test functions we need in the context of very weak solutions the space $C_{0, \sigma}^{2}(\bar{\Omega})=\left\{w \in C^{2}(\bar{\Omega}) ;\left.w\right|_{\partial \Omega}=0\right.$, $\left.\operatorname{div} w=0\right\}$; for weak instationary solutions let the space $C_{0}^{\infty}\left([0, T) ; C_{0, \sigma}^{\infty}(\Omega)\right)$ denote vector fields 
$w \in C_{0}^{\infty}([0, T) \times \Omega)$ such that $\operatorname{div}_{x} w=0$ for all $t \in[0, T)$ taking the divergence $\operatorname{div}_{x}$ with respect to $x=\left(x_{1}, x_{2}, x_{3}\right) \in \Omega$. The pairing of functions on $\Omega$ and $(0, T) \times \Omega$ is denoted by $\langle\cdot, \cdot\rangle_{\Omega}$ and $\langle\cdot, \cdot\rangle_{\Omega, T}$, respectively.

For $1 \leq q, s \leq \infty$ the usual Bochner space $L^{s}\left(0, T ; L^{q}(\Omega)\right)$ is equipped with the norm $\|\cdot\|_{q, s ; T}=\left(\int_{0}^{T}\|\cdot\|_{q}^{s} d \tau\right)^{1 / s}$ when $s<\infty$ and $\|\cdot\|_{q, \infty ; T}=$ $\operatorname{ess} \sup _{(0, T)}\|\cdot\|_{q}$ when $s=\infty$.

Let $P_{q}: L^{q}(\Omega) \rightarrow L_{\sigma}^{q}(\Omega), 1<q<\infty$, be the Helmholtz projection, and let $A_{q}=-P_{q} \Delta$ with domain $D\left(A_{q}\right)=W^{2, q}(\Omega) \cap W_{0}^{1, q}(\Omega) \cap L_{\sigma}^{q}(\Omega)$ and range $R\left(A_{q}\right)=L_{\sigma}^{q}(\Omega)$ denote the Stokes operator. We write $P=P_{q}$ and $A=A_{q}$ if there is no misunderstanding. For $-1 \leq \alpha \leq 1$ the fractional powers $A_{q}^{\alpha}: \mathcal{D}\left(A_{q}^{\alpha}\right) \rightarrow L_{\sigma}^{q}(\Omega)$ are well-defined closed operators with $\left(A_{q}^{\alpha}\right)^{-1}=A_{q}^{-\alpha}$. For $0 \leq \alpha \leq 1$ we have $D\left(A_{q}\right) \subseteq D\left(A_{q}^{\alpha}\right) \subseteq L_{\sigma}^{q}(\Omega)$ and $R\left(A_{q}^{\alpha}\right)=L_{\sigma}^{q}(\Omega)$. Then there holds the embedding estimate

$$
\|v\|_{q} \leq C\left\|A_{q}^{\alpha} v\right\|_{\gamma}, \quad 0 \leq \alpha \leq 1,2 \alpha+\frac{3}{q}=\frac{3}{\gamma}, 1<\gamma \leq q,
$$

for all $v \in D\left(A_{q}^{\alpha}\right)$. Further, we need the Stokes semigroup $e^{-t A_{q}}: L_{\sigma}^{q}(\Omega) \rightarrow$ $L_{\sigma}^{q}(\Omega), t \geq 0$, satisfying the estimate

$$
\left\|A_{q}^{\alpha} e^{-t A_{q}} v\right\|_{q} \leq C t^{-\alpha} e^{-\beta t}\|v\|_{q}, 0 \leq \alpha \leq 1, t>0,
$$

for $v \in L_{\sigma}^{q}(\Omega)$ with constants $C=C(\Omega, q, \alpha)>0, \beta=\beta(\Omega, q)>0$; for details see $[2,7,8,9,11]$.

In order to solve the perturbed system (1.6) we use an approximation procedure based on Yosida's smoothing operators

$$
J_{m}=\left(I+\frac{1}{m} A^{1 / 2}\right)^{-1} \quad \text { and } \quad \mathcal{J}_{m}=\left(I+\frac{1}{m}(-\Delta)^{1 / 2}\right)^{-1}, m \in \mathbb{N}
$$

where $I$ denotes the identity and $-\Delta$ the Dirichlet Laplacian on $\Omega$. In particular, we need the properties

$$
\begin{aligned}
& \left\|J_{m} v\right\|_{q} \leq C\|v\|_{q},\left\|A^{1 / 2} J_{m} v\right\|_{q} \leq m C\|v\|_{q}, m \in \mathbb{N}, \\
& \lim _{m \rightarrow \infty} J_{m} v=v \quad \text { for all } v \in L_{\sigma}^{q}(\Omega)
\end{aligned}
$$

and analogous results for $\mathcal{J}_{m} v, v \in L^{q}(\Omega)$; see $[8,9,16]$.

To solve the instationary Stokes system in $[0, T) \times \Omega$, cf. $[1,13,16,17,18]$, let us recall some properties for the special system 


$$
\begin{aligned}
V_{t}-\Delta V+\nabla H & =f_{0}+\operatorname{div} F_{0}, & \operatorname{div} V & =0 \\
V & =0 \text { on } \partial \Omega, & V(0) & =V_{0}
\end{aligned}
$$

with data

$$
f_{0} \in L^{1}\left(0, T ; L^{2}(\Omega)\right), F_{0} \in L^{2}\left(0, T ; L^{2}(\Omega)\right), V_{0} \in L_{\sigma}^{2}(\Omega) ;
$$
here $F_{0}=\left(F_{0, i j}\right)_{i, j=1}^{3}$ and $\operatorname{div} F_{0}=\left(\sum_{i=1}^{3} \frac{\partial}{\partial x_{i}} F_{0, i j}\right)_{j=1}^{3}$. The linear system (2.5)
admits a unique weak solution

$$
V \in L^{\infty}\left(0, T ; L_{\sigma}^{2}(\Omega)\right) \cap L^{2}\left(0, T ; W_{0}^{1,2}(\Omega)\right),
$$

satisfying the variational formulation

$$
-\left\langle V, w_{t}\right\rangle_{\Omega, T}+\langle\nabla V, \nabla w\rangle_{\Omega, T}=\left\langle V_{0}, w(0)\right\rangle_{\Omega}+\left\langle f_{0}, w\right\rangle_{\Omega, T}-\left\langle F_{0}, \nabla w\right\rangle_{\Omega, T}
$$

for all $w \in C_{0}^{\infty}\left([0, T) ; C_{0, \sigma}^{\infty}(\Omega)\right)$, and the energy equality

$$
\frac{1}{2}\|V(t)\|_{2}^{2}+\int_{0}^{t}\|\nabla V\|_{2}^{2} d \tau=\frac{1}{2}\left\|V_{0}\right\|_{2}^{2}+\int_{0}^{t}\left\langle f_{0}, V\right\rangle_{\Omega} d \tau-\int_{0}^{t}\left\langle F_{0}, \nabla V\right\rangle_{\Omega} d \tau
$$

for $0 \leq t<T$. As a consequence of (2.8) we get the energy estimate

$$
\frac{1}{2}\|V\|_{2, \infty ; T}^{2}+\|\nabla V\|_{2,2 ; T}^{2} \leq 8\left(\left\|V_{0}\right\|_{2}^{2}+\left\|f_{0}\right\|_{2,1 ; T}^{2}+\left\|F_{0}\right\|_{2,2 ; T}^{2}\right),
$$

and see that $V:[0, T) \rightarrow L_{\sigma}^{2}(\Omega)$ is continuous with $V(0)=V_{0}$. Moreover, it holds the well-defined representation formula

$$
V(t)=e^{-t A} V_{0}+\int_{0}^{t} e^{-(t-\tau) A} P f_{0} d \tau+\int_{0}^{t} A^{1 / 2} e^{-(t-\tau) A} A^{-1 / 2} P \operatorname{div} F_{0} d \tau,
$$

$0 \leq t<T$; see [16, Theorems IV.2.3.1 and 2.4.1, Lemma IV.2.4.2], and, concerning the operator $A^{-1 / 2} P$ div , [16, Ch. III.2.6].

Consider the perturbed system (1.6) with $f=\operatorname{div} F, v_{0}, k$ and $E$ as in Definition 1.1, here written in the form

$$
v_{t}-\Delta v+\operatorname{div}(v+E)(v+E)-k(v+E)+\nabla p^{*}=f, \operatorname{div} v=0
$$

together with the initial-boundary conditions $v=0$ on $\partial \Omega$ and $v(0)=v_{0}$.

In order to obtain the following approximate system, see [16, V, 2.2] for the known case $E \equiv 0$, we insert the Yosida operators (2.3) into (2.11) as follows: 


$$
\begin{aligned}
v_{t}-\Delta v+\operatorname{div}\left(J_{m} v+E\right)(v+E)-\left(\mathcal{J}_{m} k\right)(v+E)+\nabla p^{*} & =f, \operatorname{div} v=0 \\
\left.v\right|_{\partial \Omega} & =0,\left.\quad v\right|_{t=0}=v_{0}
\end{aligned}
$$

with $v=v_{m}, m \in \mathbb{N}$. Setting

$$
F_{m}(v)=\left(J_{m} v+E\right)(v+E), f_{m}(v)=\left(\mathcal{J}_{m} k\right)(v+E)
$$

we write the approximate system (2.12) in the form

$$
\begin{aligned}
& v_{t}-\Delta v+\nabla p^{*}=f_{m}(v)+\operatorname{div}\left(F-F_{m}(v)\right), \operatorname{div} v=0, \\
&\left.v\right|_{\partial \Omega}=0,\left.v\right|_{t=0}=v_{0},
\end{aligned}
$$

as a linear system, see (2.5), with right-hand side depending on $v$. In this form we use the properties (2.6)-(2.10) of the linear system (2.5).

The following definition for (2.12) is obtained similarly as Definition 1.1.

Definition 2.1. (Approximate system). Suppose

$$
\begin{aligned}
& f=\operatorname{div} F, F \in L^{2}\left(0, T ; L^{2}(\Omega)\right), v_{0} \in L_{\sigma}^{2}(\Omega), \\
& E \in L^{s}\left(0, T ; L^{q}(\Omega)\right), \operatorname{div} E=k \in L^{4}\left(0, T ; L^{2}(\Omega)\right), \\
& 4 \leq s<\infty, 4 \leq q<\infty, \frac{2}{s}+\frac{3}{q}=1 .
\end{aligned}
$$

Then a vector field $v=v_{m}, m \in \mathbb{N}$, is called a weak solution of the approximate system $(2.12)$ in $[0, T) \times \Omega$ with data $f$, $v_{0}$ if the following conditions are satisfied:

a)

$$
v \in L_{\mathrm{loc}}^{\infty}\left([0, T) ; L_{\sigma}^{2}(\Omega)\right) \cap L_{\mathrm{loc}}^{2}\left([0, T) ; W_{0}^{1,2}(\Omega)\right)
$$

b) for each $w \in C_{0}^{\infty}\left([0, T) ; C_{0, \sigma}^{\infty}(\Omega)\right)$,

$$
\begin{gathered}
-\left\langle v, w_{t}\right\rangle_{\Omega, T}+\langle\nabla v, \nabla w\rangle_{\Omega, T}-\left\langle\left(J_{m} v+E\right)(v+E), \nabla w\right\rangle_{\Omega, T} \\
-\left\langle\left(\mathcal{J}_{m} k\right)(v+E), w\right\rangle_{\Omega, T}=\left\langle v_{0}, w(0)\right\rangle_{\Omega}-\langle F, \nabla w\rangle_{\Omega, T},
\end{gathered}
$$

c) for $0 \leq t<T$,

$$
\frac{1}{2}\|v(t)\|_{2}^{2}+\int_{0}^{t}\|\nabla v\|_{2}^{2} d \tau \leq \frac{1}{2}\left\|v_{0}\right\|_{2}^{2}-\int_{0}^{t}\left\langle F-\left(J_{m} v+E\right) E, \nabla v\right\rangle_{\Omega} d \tau
$$

$$
+\int_{0}^{t}\left\langle\left(\mathcal{J}_{m} k-\frac{1}{2} k\right) v, v\right\rangle_{\Omega} d \tau+\int_{0}^{t}\left\langle\left(\mathcal{J}_{m} k\right) E, v\right\rangle_{\Omega} d \tau,
$$

d) $v:[0, T) \rightarrow L_{\sigma}^{2}(\Omega)$ is continuous satisfying $v(0)=v_{0}$. 


\section{The approximate system.}

The following existence result yields a weak solution $v=v_{m}$ of (2.12) first of all only in an interval $\left[0, T^{\prime}\right)$ where $T^{\prime}=T^{\prime}(m)>0$ is sufficiently small.

Lemma 3.1. Let $f, k, E, v_{0}$ be as in Definition 2.1 and let $m \in \mathbb{N}$. Then there exists some $T^{\prime}=T^{\prime}\left(f, k, E, v_{0}, m\right), 0<T^{\prime} \leq \min (1, T)$, such that the approximate system (2.12) has a unique weak solution $v=v_{m}$ in $\left[0, T^{\prime}\right) \times \Omega$ with data $f, v_{0}$ in the sense of Definition 2.1 with $T$ replaced by $T^{\prime \prime}$.

Proof. First we consider a given weak solution $v=v_{m}$ of (2.12) in $\left[0, T^{\prime}\right) \times \Omega$ with any $0<T^{\prime} \leq 1$. Hence it holds

$$
v \in X_{T^{\prime}}:=L^{\infty}\left(0, T^{\prime} ; L_{\sigma}^{2}(\Omega)\right) \cap L^{2}\left(0, T^{\prime} ; W_{0}^{1,2}(\Omega)\right)
$$

with

$$
\|v\|_{X_{T^{\prime}}}:=\|v\|_{2, \infty ; T^{\prime}}+\left\|A^{\frac{1}{2}} v\right\|_{2,2 ; T^{\prime}}<\infty .
$$

Using Hölder's inequality and several embedding estimates, see [16, Ch. V.1.2], we obtain with some constant $C=C(\Omega)>0$ the estimates

$$
\begin{aligned}
\left\|\left(J_{m} v\right) v\right\|_{2,2 ; T^{\prime}} & \leq C\left\|J_{m} v\right\|_{6,4 ; T^{\prime}}\|v\|_{3,4 ; T^{\prime}} \\
& \leq C\left\|A^{1 / 2} J_{m} v\right\|_{2,4 ; T^{\prime}}\|v\|_{X_{T^{\prime}}} \\
& \leq C m\|v\|_{2,4 ; T^{\prime}} \leq C m\left(T^{\prime}\right)^{1 / 4}\|v\|_{X_{T^{\prime}}}^{2},
\end{aligned}
$$

and

$$
\begin{aligned}
\left\|\left(J_{m} v\right) E\right\|_{2,2 ; T^{\prime}} & \leq C\left\|J_{m} v\right\|_{4,4 ; T^{\prime}}\|E\|_{4,4 ; T^{\prime}} \leq C\left\|J_{m} v\right\|_{6,4 ; T^{\prime}}\|E\|_{4,4 ; T^{\prime}} \\
& \leq C m\left(T^{\prime}\right)^{1 / 4}\|v\|_{X_{T^{\prime}}}\|E\|_{4,4 ; T^{\prime}}, \\
\|E v\|_{2,2 ; T^{\prime}} & \leq C\|E\|_{q, s ; T^{\prime}}\|v\|_{\left(\frac{1}{2}-\frac{1}{q}\right)^{-1},\left(\frac{1}{2}-\frac{1}{8}\right)^{-1}, T^{\prime}} \leq C\|E\|_{q, s ; T^{\prime}}\|v\|_{X_{T^{\prime}}}
\end{aligned}
$$

of course, $\|E E\|_{2,2 ; T^{\prime}} \leq C\|E\|_{4,4 ; T^{\prime}}^{2}$. Moreover,

$$
\begin{aligned}
(3.5)\left\|\left(\mathcal{J}_{m} k\right) v\right\|_{2,1 ; T^{\prime}} & \leq C\left\|\mathcal{J}_{m} k\right\|_{3,2 ; T^{\prime}}\|v\|_{6,2 ; T^{\prime}} \leq C\left\|(-\Delta)^{\frac{1}{2}} \mathcal{J}_{m} k\right\|_{2,2 ; T^{\prime}}\|v\|_{X_{T^{\prime}}} \\
& \leq C m\|k\|_{2,2 ; T^{\prime}}\|v\|_{X_{T^{\prime}}} \leq C m\left(T^{\prime}\right)^{\frac{1}{4}}\|k\|_{2,4 ; T^{\prime}}\|v\|_{X_{T^{\prime}}} \\
\text { (3.6) }\left\|\left(\mathcal{J}_{m} k\right) E\right\|_{2,1 ; T^{\prime}} & \leq C\left\|\mathcal{J}_{m} k\right\|_{4,2 ; T^{\prime}}\|E\|_{4,2 ; T^{\prime}} \leq C\left\|(-\Delta)^{\frac{1}{2}} \mathcal{J}_{m} k\right\|_{2,2 ; T^{\prime}}\|E\|_{4,4 ; T^{\prime}} \\
& \leq C m\|k\|_{2,2 ; T^{\prime}}\|E\|_{4,4 ; T^{\prime}} \leq C m\left(T^{\prime}\right)^{\frac{1}{4}}\|k\|_{2,4 ; T^{\prime}}\|E\|_{4,4 ; T^{\prime}}
\end{aligned}
$$


Using (2.14) and the energy estimate (2.9) with $f_{0}, F_{0}$ replaced by $f_{m}(v)$, $F-F_{m}(v)$ we get from (3.2)-(3.5) the estimate

$$
\begin{aligned}
\|v\|_{X_{T^{\prime}}} \leq C & \left(\left\|v_{0}\right\|_{2}+\|F\|_{2,2 ; T^{\prime}}+\|E\|_{4,4 ; T^{\prime}}^{2}+m\left(T^{\prime}\right)^{\frac{1}{4}}\|v\|_{X_{T^{\prime}}}^{2}+\right. \\
& +m\left(T^{\prime}\right)^{\frac{1}{4}}\|v\|_{X_{T^{\prime}}}\|E\|_{4,4 ; T^{\prime}}+\|v\|_{X_{T^{\prime}}}\|E\|_{q, s ; T^{\prime}}+ \\
& \left.+m\left(T^{\prime}\right)^{\frac{1}{4}}\|k\|_{2,4 ; T^{\prime}}\left(\|E\|_{4,4 ; T^{\prime}}+\|v\|_{X_{T^{\prime}}}\right)\right)
\end{aligned}
$$

with $C=C(\Omega)>0$.

Applying (2.10) to (2.14) we obtain the equation

$$
v=\mathcal{F}_{T^{\prime}}(v)
$$

where

$$
\begin{aligned}
\left(\mathcal{F}_{T^{\prime}}(v)\right)(t)=e^{-t A} & v_{0}+\int_{0}^{t} e^{-(t-\tau) A} \operatorname{Pf}_{m}(v) d \tau \\
& +\int_{0}^{t} A^{\frac{1}{2}} e^{-(t-\tau) A} A^{-\frac{1}{2}} P \operatorname{div}\left(F-F_{m}(v)\right) d \tau .
\end{aligned}
$$

Let

$$
\begin{aligned}
& a=C m\left(T^{\prime}\right)^{\frac{1}{4}}, b=C\|E\|_{q, s ; T^{\prime}}+C m\left(T^{\prime}\right)^{\frac{1}{4}}\|E\|_{4,4 ; T^{\prime}}+C m\left(T^{\prime}\right)^{\frac{1}{4}}\|k\|_{2,4 ; T^{\prime}}, \\
& d=C\left(\left\|v_{0}\right\|_{2}+\|E\|_{4,4 ; T^{\prime}}^{2}+\|F\|_{2,2 ; T^{\prime}}+m\left(T^{\prime}\right)^{\frac{1}{4}}\|k\|_{2,4 ; T^{\prime}}\|E\|_{4,4 ; T^{\prime}}\right)
\end{aligned}
$$

with $C$ as in (3.7). Then (3.7) may be rewritten in the form

$$
\left\|\mathcal{F}_{T^{\prime}}(v)\right\|_{X_{T^{\prime}}} \leq a\|v\|_{X_{T^{\prime}}}^{2}+b\|v\|_{X_{T^{\prime}}}+d .
$$

Up to now $v=v_{m}$ was a given solution as desired in Lemma 3.1. In the next step we treat (3.8) as a fixed point equation in $X_{T^{\prime}}$ and show with Banach's fixed point principle that (3.8) has a solution $v=v_{m}$ if $T^{\prime}>0$ is sufficiently small.

Thus let $v \in X_{T^{\prime}}$ and choose $0<T^{\prime} \leq \min (1, T)$ such that the smallness condition

$$
4 a d+2 b<1
$$

is satisfied. Then the quadratic equation $y=a y^{2}+b y+d$ has a minimal positive root given by

$$
0<y_{1}=2 d\left(1-b+\sqrt{b^{2}+1-(4 a d+2 b)}\right)^{-1}<2 d
$$


and, since $y_{1}=a y_{1}^{2}+b y_{1}+d>d$, we conclude that $\mathcal{F}_{T^{\prime}}$ maps the closed ball $B_{T^{\prime}}=\left\{v \in X_{T^{\prime}}:\|v\|_{X_{T^{\prime}}} \leq y_{1}\right\}$ into itself.

Further let $v_{1}, v_{2} \in B_{T^{\prime}}$. Then we obtain similarly as in (3.10) the estimate

$$
\begin{aligned}
& \left\|\mathcal{F}_{T^{\prime}}\left(v_{1}\right)-\mathcal{F}_{T^{\prime}}\left(v_{2}\right)\right\|_{X_{T^{\prime}}} \leq C m\left(T^{\prime}\right)^{\frac{1}{4}}\left\|v_{1}-v_{2}\right\|_{X_{T^{\prime}}}\left(\left\|v_{1}\right\|_{X_{T^{\prime}}}+\left\|v_{2}\right\|_{X_{T^{\prime}}}\right) \\
& \quad+C\left\|v_{1}-v_{2}\right\|_{X_{T^{\prime}}}\left(\|E\|_{q, s ; T^{\prime}}+m\left(T^{\prime}\right)^{\frac{1}{4}}\|k\|_{2,4 ; T^{\prime}}+m\left(T^{\prime}\right)^{\frac{1}{4}}\|E\|_{4,4 ; T^{\prime}}\right) \\
& \quad \leq\left\|v_{1}-v_{2}\right\|_{X_{T^{\prime}}}\left(a\left(\left\|v_{1}\right\|_{X_{T^{\prime}}}+\left\|v_{2}\right\|_{X_{T^{\prime}}}\right)+b\right)
\end{aligned}
$$

where

$$
a\left(\left\|v_{1}\right\|_{X_{T^{\prime}}}+\left\|v_{2}\right\|_{X_{T^{\prime}}}\right)+b \leq 2 a y_{1}+b<4 a d+2 b<1 .
$$

This means that $\mathcal{F}_{\mathcal{T}^{\prime}}$ is a strict contraction on $B_{T^{\prime}}$. Now Banach's fixed point principle yields a solution $v=v_{m} \in B_{T^{\prime}}$ of (3.8) which is unique in $B_{T^{\prime}}$.

Using (2.6)-(2.10) with $f_{0}+\operatorname{div} F_{0}$ replaced by $f_{m}(v)+\operatorname{div}\left(F-F_{m}(v)\right)$ we conclude from (3.8) that $v=v_{m}$ is a solution of the approximate system (2.12) in the sense of Definition 2.1.

Finally we show that $v$ is unique not only in $B_{T^{\prime}}$, but even in the whole space $X_{T^{\prime}}$. Indeed, consider any solution $\tilde{v} \in X_{T^{\prime}}$ of (2.12). Then there exists some $0<T^{*} \leq \min \left(1, T^{\prime}\right)$ such that $\|\tilde{v}\|_{X_{T^{*}}} \leq y_{1}$, and using (3.12), (3.13) with $v_{1}, v_{2}$ replaced by $v, \tilde{v}$ we conclude that $v=\tilde{v}$ on $\left[0, T^{*}\right]$. When $T^{*}<T^{\prime}$ we repeat this step finitely many times and obtain that $v=\tilde{v}$ on $\left[0, T^{\prime}\right)$. This completes the proof of Lemma 3.1.

The next preliminary result yields an energy estimate for the approximate solution $v=v_{m}$ of (2.12). It is important that the right-hand side of this estimate does not depend on $m \in \mathbb{N}$. This will enable us to treat the limit $m \rightarrow \infty$ and to get the desired solution in Theorem 1.4, a).

Lemma 3.2. Consider any weak solution $v=v_{m}, m \in \mathbb{N}$, of the approximate system (2.12) in the sense of Definition 2.1. Then there is a constant $C=C(\Omega)>0$ such that the energy estimate

$$
\begin{aligned}
& \|v(t)\|_{2}^{2}+\int_{0}^{t}\|\nabla v\|_{2}^{2} d \tau \\
& \leq C\left(\left\|v_{0}\right\|_{2}^{2}+\|F\|_{2,2 ; t}^{2}+\|k\|_{2,4 ; t}^{4}+\|E\|_{4,4 ; t}^{4}\right) \exp \left(C\|k\|_{2,4 ; t}^{4}+C\|E\|_{q, s ; t}^{s}\right)
\end{aligned}
$$

holds for $0 \leq t<T$. 
Proof. The proof of (3.14) is based on the energy inequality (2.18). Using similar arguments as in (3.2)-(3.6) we obtain the following estimates of the right-hand side terms in (2.18); here $\varepsilon>0$ means an absolute constant, $C_{0}=C_{0}(\Omega)>0$ and $C=C(\varepsilon, \Omega)>0$ do not depend on $m$, and $\alpha=\frac{2}{s}=1-\frac{3}{q}$. First of all

$$
\begin{aligned}
\left|\int_{0}^{t}\left\langle\left(J_{m} v\right) E, \nabla v\right\rangle_{\Omega} d \tau\right| & \leq C_{0} \int_{0}^{t}\left\|J_{m} v\right\|_{\left(\frac{1}{2}-\frac{1}{q}\right)^{-1}}\|E\|_{q}\|\nabla v\|_{2} d \tau \\
& \leq C_{0} \int_{0}^{t}\|v\|_{\left(\frac{1}{2}-\frac{1}{q}\right)^{-1}}\|E\|_{q}\|\nabla v\|_{2} d \tau \\
& \leq C_{0} \int_{0}^{t}\|v\|_{2}^{\alpha}\|E\|_{q}\|\nabla v\|_{2}^{2-\alpha} d \tau \\
& \leq \varepsilon\|\nabla v\|_{2,2 ; t}^{2}+C \int_{0}^{t}\|E\|_{q}^{s}\|v\|_{2}^{2} d \tau
\end{aligned}
$$

and

$$
\begin{aligned}
& \left|\int_{0}^{t}\langle E E, \nabla v\rangle_{\Omega} d \tau\right| \leq C_{0} \int_{0}^{t}\|E\|_{4}^{2}\|\nabla v\|_{2} d \tau \leq \varepsilon\|\nabla v\|_{2,2 ; t}^{2}+C\|E\|_{4,4 ; t}^{4}, \\
& \left|\int_{0}^{t}\langle F, \nabla v\rangle_{\Omega} d \tau\right| \leq \varepsilon\|\nabla v\|_{2,2 ; t}^{2}+C\|F\|_{2,2 ; t}^{2} .
\end{aligned}
$$

Moreover, since $\|v\|_{4} \leq C_{0}\|\nabla v\|_{2}^{1 / 4}\|\nabla v\|_{2}^{3 / 4}$,

$$
\begin{aligned}
\left|\int_{0}^{t}\left\langle\mathcal{J}_{m} k v, v\right\rangle_{\Omega} d \tau\right| & \leq \varepsilon\|\nabla v\|_{2,2 ; t}^{2}+C \int_{0}^{t}\|k\|_{2}^{4}\|v\|_{2}^{2} d \tau, \\
\left|\int_{0}^{t}\left\langle\left(\mathcal{J}_{m} k\right) E, v\right\rangle_{\Omega} d \tau\right| & \leq C_{0} \int_{0}^{t}\left\|\left(\mathcal{J}_{m} k\right) E\right\|_{\frac{6}{5}}\|v\|_{6} d \tau \\
& \leq C_{0} \int_{0}^{t}\|k\|_{2}\|E\|_{3}\|\nabla v\|_{2} d \tau \\
& \leq \varepsilon\|\nabla v\|_{2,2 ; t}^{2}+C\left(\|k\|_{2,4 ; t}^{4}+\|E\|_{4,4 ; t}^{4}\right) .
\end{aligned}
$$


A similar estimate as for $\int_{0}^{t}\left\langle\mathcal{J}_{m} k v, v\right\rangle_{\Omega} d \tau$ also holds for $\int_{0}^{t}\langle k v, v\rangle_{\Omega} d \tau$.

Choosing $\varepsilon>0$ sufficiently small we apply these inequalities to (2.18) and obtain that

$$
\begin{gathered}
\|v(t)\|_{2}^{2}+\|\nabla v\|_{2,2 ; t}^{2} \leq C\left(\left\|v_{0}\right\|_{2}^{2}+\|F\|_{2,2 ; t}^{2}+\|E\|_{4,4 ; t}^{4}+\|k\|_{2,4 ; t}^{4}\right) \\
+C \int_{0}^{t}\left(\|k\|_{2}^{4}+\|E\|_{q}^{s}\right)\|v\|_{2}^{2} d \tau
\end{gathered}
$$

for $0 \leq t<T$. Then Gronwall's lemma implies that

$$
\begin{aligned}
& \|v(t)\|_{2}^{2}+\int_{0}^{t}\|\nabla v\|_{2}^{2} d \tau \leq C\left(\left\|v_{0}\right\|_{2}^{2}+\|F\|_{2,2 ; t}^{2}+\|E\|_{4,4 ; t}^{4}+\|k\|_{2,4 ; t}^{4}\right) \\
& \times \exp \left(C\|k\|_{2,4 ; t}^{4}+C\|E\|_{q, s ; t}^{s}\right)
\end{aligned}
$$

for $0 \leq t<T$. This yields the estimate (3.14).

The next result proves the existence of a unique approximate solution $v=v_{m}$ for the given interval $[0, T)$.

Lemma 3.3. Let $f, k, E, v_{0}$ be given as in Definition 2.1 and let $m \in \mathbb{N}$. Then there exists a unique weak solution $v=v_{m}$ of the approximate system (2.12) in $[0, T) \times \Omega$ with data $f, v_{0}$.

Proof. Lemma 3.1 yields such a solution if $0<T \leq 1$ is sufficiently small. Let $\left[0, T^{*}\right) \subseteq[0, T), T^{*}>0$, be the largest interval of existence of such a solution $v=v_{m}$ in $\left[0, T^{*}\right) \times \Omega$, and assume that $T^{*}<T$. Further we choose some finite $T^{* *}>T^{*}$ with $T^{* *} \leq T$, and some $T_{0}$ satisfying $0<T_{0}<T^{*}$. Then we apply Lemma 3.1 with $\left[0, T^{\prime}\right)$ replaced by $\left[T_{0}, T_{0}+\delta\right.$ ) where $\delta>0, T_{0}+\delta \leq T^{* *}$, and find a unique weak solution $v^{*}=v_{m}^{*}$ of the system (2.12) in $\left[T_{0}, T_{0}+\delta\right) \times \Omega$ with initial value $\left.v^{*}\right|_{t=T_{0}}=v\left(T_{0}\right)$. The length $\delta$ of the existence interval $\left[T_{0}, T_{0}+\delta\right.$ ), see the proof of Lemma 3.1, only depends on $\left\|v\left(T_{0}\right)\right\|_{2} \leq\|v\|_{2, \infty ; T^{*}}<\infty$ and on $\|F\|_{2,2 ; T^{* *}},\|E\|_{q, s ; T^{* *}}$, $\|k\|_{2,4: T^{* *}}$, and can be chosen independently of $T_{0}$. Therefore, we can choose $T_{0}$ close to $T^{*}$ in such a way that $T^{*}<T_{0}+\delta \leq T^{* *}$. Then $v^{*}$ yields a unique extension of $v$ from $\left[0, T^{*}\right)$ to $\left[0, T_{0}+\delta\right)$ which is a contradiction. This proves the lemma. 
In the next step, see $\S 4$ below, we are able to let $m \rightarrow \infty$ similarly as in the classical case $E \equiv 0$. This will yield a solution of the perturbed system (1.6).

\section{Proof of Theorem 1.4.}

It is sufficient to prove Theorem 1.4, a). For this purpose we start with the sequence $\left(v_{m}\right)$ of solutions of the approximate system (2.12) constructed in Lemma 3.3. Then, using Lemma 3.2, we find for each finite $T^{*}, 0<T^{*} \leq T$, some constant $C_{T^{*}}>0$ not depending on $m$ such that

$$
\left\|v_{m}\right\|_{2, \infty ; T^{*}}^{2}+\left\|\nabla v_{m}\right\|_{2,2 ; T^{*}}^{2} \leq C_{T^{*}} .
$$

Hence there exists a vector field

$$
v \in L^{\infty}\left(0, T^{*} ; L_{\sigma}^{2}(\Omega)\right) \cap L^{2}\left(0, T^{*} ; W_{0}^{1,2}(\Omega)\right),
$$

and a subsequence of $\left(v_{m}\right)$, for simplicity again denoted by $\left(v_{m}\right)$, with the following properties, see, e.g. [16, Ch. V.3.3]:

$$
\begin{aligned}
& v_{m} \rightarrow v \text { in } L^{2}\left(0, T^{*} ; W_{0}^{1,2}(\Omega)\right) \quad \text { (weakly) } \\
& v_{m} \rightarrow v \text { in } L^{2}\left(0, T^{*} ; L^{2}(\Omega)\right) \quad(\text { strongly) } \\
& v_{m}(t) \rightarrow v(t) \text { in } L^{2}(\Omega) \text { for a.a. } t \in\left[0, T^{*}\right) .
\end{aligned}
$$

Moreover, for all $t \in\left[0, T^{*}\right)$ we obtain that

$$
\begin{aligned}
\|\nabla v\|_{2,2 ; t}^{2} & \leq \liminf _{m \rightarrow \infty}\left\|\nabla v_{m}\right\|_{2,2 ; t}^{2}, \\
\|v(t)\|_{2}^{2} & \leq \liminf _{m \rightarrow \infty}\left\|v_{m}(t)\right\|_{2}^{2} .
\end{aligned}
$$

Further, using Hölder's inequality and (4.2)-(4.4) we get with some further subsequence, again denoted by $\left(v_{m}\right)$, that

$$
\begin{gathered}
v_{m} \rightarrow v \quad \text { in } L^{s_{1}}\left(0, T^{*} ; L^{q_{1}}(\Omega)\right), \frac{2}{s_{1}}+\frac{3}{q_{1}}=\frac{3}{2}, 2 \leq s_{1}, q_{1}<\infty, \\
(4.5) \quad v_{m} v_{m} \rightarrow v v \quad \text { in } L^{s_{2}}\left(0, T^{*} ; L^{q_{2}}(\Omega)\right), \frac{2}{s_{2}}+\frac{3}{q_{2}}=3,1 \leq s_{2}, q_{2}<\infty, \\
v_{m} \cdot \nabla v_{m} \rightarrow v \cdot \nabla v \quad \text { in } L^{s_{3}}\left(0, T^{*} ; L^{q_{3}}(\Omega)\right), \frac{2}{s_{3}}+\frac{3}{q_{3}}=4,1 \leq s_{3}, q_{3}<\infty,
\end{gathered}
$$


and that with some constant $C=C_{T^{*}}>0$ :

$$
\begin{aligned}
&\left\|\left(J_{m} v_{m}\right) v_{m}\right\|_{q_{2}, s_{2} ; T^{*}} \leq C\left\|v_{m}\right\|_{q_{1}, s_{1} ; T^{*}}^{2} \\
&\left\|\left(J_{m} v_{m}\right) E\right\|_{\left(\frac{1}{q}+\frac{1}{q_{1}}\right)^{-1},\left(\frac{1}{s}+\frac{1}{s_{1}}\right)^{-1} ; T^{*}} \leq C\left\|v_{m}\right\|_{q_{1}, s_{1} ; T^{*}}\|E\|_{q, s ; T^{*}} \\
&\left\|E v_{m}\right\|_{\left(\frac{1}{q}+\frac{1}{q_{1}}\right)^{-1},\left(\frac{1}{s}+\frac{1}{s_{1}}\right)^{-1} ; T^{*}} \leq C\left\|v_{m}\right\|_{q_{1}, s_{1} ; T^{*}}\|E\|_{q, s ; T^{*}} \\
&\left|\left\langle\left(J_{m} v_{m}\right) E, \nabla v_{m}\right\rangle_{\Omega, T^{*}}\right| \leq C\left\|v_{m}\right\|_{q_{1}, s_{1} ; T^{*}}\|E\|_{q, s ; T^{*}}\left\|\nabla v_{m}\right\|_{2,2 ; T^{*}}
\end{aligned}
$$

as well as

$$
\begin{aligned}
\left|\left\langle k v_{m}, v_{m}\right\rangle_{\Omega, T^{*}}\right| & \leq C\|k\|_{2,4 ; T^{*}}\left\|v_{m}\right\|_{q_{1}, s_{1} ; T^{*}}^{2} \\
\left|\left\langle\left(\mathcal{J}_{m} k\right) v_{m}, v_{m}\right\rangle_{\Omega, T^{*}}\right| & \leq C\|k\|_{2,4 ; T^{*}}\left\|v_{m}\right\|_{q_{1}, s_{1} ; T^{*}}^{2} \\
\left|\left\langle\left(\mathcal{J}_{m} k\right) E, v_{m}\right\rangle_{\Omega, T^{*}}\right| & \leq C\|k\|_{2,4 ; T^{*}}\|E\|_{q, s ; T^{*}}\left\|v_{m}\right\|_{q_{1}, s_{1} ; T^{*}}
\end{aligned}
$$

The theorem is proved when we show that (2.16)-(2.18) imply letting $m \rightarrow \infty$ the properties (1.8)-(1.10) and the estimate (1.28). This proof rests on the above arguments (4.1)-(4.10).

Obviously, (1.8) follows from (4.1), letting $m \rightarrow \infty$. Further, the relation (1.9) follows from (2.17) and (2.4) using that

$$
\begin{aligned}
\left\langle v_{m}, w_{t}\right\rangle_{\Omega, T^{*}} & \rightarrow\left\langle v, w_{t}\right\rangle_{\Omega, T^{*}} \\
\left\langle\nabla v_{m}, \nabla w\right\rangle_{\Omega, T^{*}} & \rightarrow\langle\nabla v, \nabla w\rangle_{\Omega, T^{*}} \\
\left\langle\left(J_{m} v_{m}+E\right)\left(v_{m}+E\right), \nabla w\right\rangle_{\Omega, T^{*}} & \rightarrow\langle(v+E)(v+E), \nabla w\rangle_{\Omega, T^{*}} \\
\left\langle\left(\mathcal{J}_{m} k\right)\left(v_{m}+E\right), w\right\rangle_{\Omega, T^{*}} & \rightarrow\langle k(v+E), w\rangle_{\Omega, T^{*}}
\end{aligned}
$$

To prove the energy inequality (1.10) we need in (2.18), letting $m \rightarrow \infty$, the following arguments.

The left-hand side of (1.10) follows obviously from (4.4). To prove the right-hand side limit $m \rightarrow \infty$ in (2.18) we first show that

$$
\left\langle\left(J_{m} v_{m}\right) E, \nabla v_{m}\right\rangle_{\Omega, T^{*}} \rightarrow\langle v E, \nabla v\rangle_{\Omega, T^{*}}
$$

It is sufficient to prove (4.12) with $E$ replaced by some smooth vector field $\tilde{E}$ such that $\|E-\tilde{E}\|_{q, s ; T^{*}}$ is sufficiently small. This follows using (4.9) with $E$ replaced by $E-\tilde{E}$. Thus we may assume in the following that $E$ in (4.12) is a smooth function $E \in C_{0}^{\infty}\left(\left[0, T^{*}\right) ; C_{0}^{\infty}(\Omega)\right)$. Using (4.1)-(4.4) and (2.4), we conclude that 


$$
\begin{aligned}
& \left|\left\langle\left(J_{m} v_{m}\right) E-v E, \nabla v_{m}\right\rangle_{\Omega, T^{*}}\right| \\
& \quad \leq\left\|\left(J_{m} v_{m}\right) E-v E\right\|_{2,2 ; T^{*}}\left\|\nabla v_{m}\right\|_{2,2 ; T^{*}} \\
& \quad \leq C(E)\left\|J_{m} v_{m}-v\right\|_{2,2 ; T^{*}} \\
& \quad \leq C(E)\left(\left\|J_{m}\left(v_{m}-v\right)\right\|_{2,2 ; T^{*}}+\left\|\left(J_{m}-I\right) v\right\|_{2,2 ; T^{*}}\right) \\
& \quad \leq C(E)\left(\left\|v_{m}-v\right\|_{2,2 ; T^{*}}+\left\|\left(J_{m}-I\right) v\right\|_{2,2 ; T^{*}}\right) \rightarrow 0
\end{aligned}
$$

as $m \rightarrow \infty$ where $C(E)>0$ is a constant. This yields (4.12).

Similarly, approximating $k$ by a smooth function $k \in C_{0}^{\infty}\left(\left[0, T^{*}\right) ; C_{0}^{\infty}(\Omega)\right)$, we obtain the convergence properties

$$
\begin{aligned}
\left\langle k v_{m}, v_{m}\right\rangle_{\Omega, T^{*}} & \rightarrow\langle k v, v\rangle_{\Omega, T^{*}}, \\
\left\langle\left(\mathcal{J}_{m} k\right) v_{m}, v_{m}\right\rangle_{\Omega, T^{*}} & \rightarrow\langle k v, v\rangle_{\Omega, T^{*}}, \\
\left\langle\left(\mathcal{J}_{m} k\right) E, v_{m}\right\rangle_{\Omega, T^{*}} & \rightarrow\langle k E, v\rangle_{\Omega, T^{*}} .
\end{aligned}
$$

Since $E \in L^{4}\left(0, T^{*} ; L^{4}(\Omega)\right)$, the convergence $\left\langle E E, \nabla v_{m}\right\rangle_{\Omega, T^{*}} \rightarrow\langle E E, \nabla v\rangle_{\Omega, T^{*}}$ is obvious.

This proves that $v$ is a weak solution in the sense of Definition 1.1.

To prove the energy estimate (1.28) we apply (4.4) to (3.14). This completes the proof.

\section{More general weak solutions.}

The existence of a weak solution $v$ for the perturbed system (1.6) under the general assumption on $E$ in Theorem 1.4 a) enables us to extend the solution class of the Navier-Stokes system (1.1) using certain generalized data. For simplicity we only consider the case $k=0$.

THEOREM 5.1 (More general weak solutions). Consider

$$
\begin{aligned}
& f=\operatorname{div} F, F \in L^{2}\left(0, T ; L^{2}(\Omega)\right), v_{0} \in L_{\sigma}^{2}(\Omega), \\
& E \in L^{s}\left(0, T ; L^{q}(\Omega)\right), 4 \leq s<\infty, 4 \leq q<\infty, \frac{2}{s}+\frac{3}{q}=1,
\end{aligned}
$$

satisfying

$$
E_{t}-\Delta E+\nabla h=0, \operatorname{div} E=0
$$

in $(0, T) \times \Omega$ in the sense of distributions with an associated pressure $h$. 
Let $v$ be a weak solution of the perturbed system (1.6) in $[0, T) \times \Omega$ in the sense of Definition 1.1 with $E, f, v_{0}$ from (5.1)-(5.3).

Then the vector field $u=v+E$ is a solution of the Navier-Stokes system

$$
\begin{aligned}
u_{t}-\Delta u+u \cdot \nabla u+\nabla p & =f, \operatorname{div} u=0 \\
\left.u\right|_{\partial \Omega} & =g,\left.u\right|_{t=0}=u_{0}
\end{aligned}
$$

in $[0, T) \times \Omega$ with external force $f$ and (formally) given data

$$
g:=\left.E\right|_{\partial \Omega}, \quad u_{0}:=v_{0}+\left.E\right|_{t=0},
$$

in the generalized (well-defined) sense that

$$
\left.(u-E)\right|_{\partial \Omega}=0,\left.\quad(u-E)\right|_{t=0}=v_{0},
$$

and (5.4) is satisfied in the sense of distributions with an associated pressure $p$.

\section{REMARK 5.2. (Regularity properties)}

a) Let $E$ in (5.2) be regular in the sense that $g$ and $E_{0}=\left.E\right|_{t=0}$ in (5.6) have the properties in Lemma 1.2. Then the solution $u=v+E$ has the properties in Theorem 1.4, b).

b) Let $E$ in (5.2) be regular in the sense that $g$ and $E_{0}=\left.E\right|_{t=0}$ in (5.6) have the properties in (1.26). Then the solution $u=v+E$ is correspondingly regular and (5.5) is well-defined in the usual strong sense.

\section{REFERENCES}

[1] H. Amann, On the strong solvability of the Navier-Stokes equations. J. Math. Fluid Mech., 2 (2000), pp. 16-98.

[2] H. Amann, Navier-Stokes equations with nonhomogeneous Dirichlet data, J. Nonlinear Math. Phys., 10, Suppl. 1 (2003), pp. 1-11.

[3] R. FARwig - G. P. Galdi - H. Sohr, A new class of weak solutions of the Navier-Stokes equations with nonhomogeneous data, J. Math. Fluid Mech., 8 (2006), pp. 423-444.

[4] R. FARWIG - H. Kozono - H. SoHR, Global weak solutions of the Navier-Stokes system with nonzero boundary conditions. Funkcial. Ekvac., 53 (2010), pp. 231-247.

[5] R. FARWIG - H. Kozono - H. SoHR, Global Leray-Hopf weak solutions of the Navier-Stokes equations with nonzero time-dependent boundary values. Progress Nonl. Differential Equations Appl., Birkhäuser-Verlag Basel, 60 (2011), pp. 211-232. 
[6] A. V. Fursikov - M. D. GunzBurger - L. S. Hou, Inhomogeneous boundary value problems for the three-dimensional evolutionary Navier-Stokes equations. J. Math. Fluid Mech., 4 (2002), pp. 45-75.

[7] G. P. GaLdi, An Introduction to the Mathematical Theory of the NavierStokes Equations. Vol. I, Springer Verlag, New York, 1994.

[8] Y. GiGA, Analyticity of the semigroup generated by the Stokes operator in $L_{r^{-}}$ spaces. Math. Z., 178 (1981), pp. 297-329.

[9] Y. Giga - H. SoHR, Abstract $L^{p}$ estimates for the Cauchy problem with applications to the Navier-Stokes equations in exterior domains. J. Funct. Anal., 102 (1991), pp. 72-94.

[10] G. GRubB, Nonhomogeneous Dirichlet Navier-Stokes problems in low regularity $L_{p}$ Sobolev spaces. J. Math. Fluid Mech., 3 (2001), pp. 57-81.

[11] J. G. HEYwood, The Navier-Stokes Equations: On the existence, regularity and decay of solutions. Indiana Univ. Math. J., 29 (1980), pp. 639-681.

[12] E. HopF, Über die Anfangswertaufgabe für die hydrodynamischen Grundgleichungen. Math. Nachr., 4 (1950), pp. 213-231.

[13] O. A. Ladyzhenskaya, The Mathematical Theory of Viscous Incompressible Flow. Gordon and Breach, New York, 1969.

[14] J. LeraY, Sur le mouvement d'un liquide visqueux emplissant l' espace. Acta Math., 63 (1934), pp. 193-248.

[15] J. P. RAYMOND, Stokes and Navier-Stokes equations with nonhomogeneous boundary conditions. Ann. Inst. Henri Poincaré, Anal. Non Linéaire 24 (2007), pp. 921-951.

[16] H. SoHR, The Navier-Stokes Equations. Birkhäuser Verlag, Basel, 2001.

[17] V. A. SolonNIKov, Estimates for solutions of nonstationary Navier-Stokes equations. J. Soviet Math., 8 (1977), pp. 467-529.

[18] R. Temam, Navier-Stokes Equations. North-Holland, Amsterdam, 1977.

Manoscritto pervenuto in redazione l'8 luglio 2010. 